\section{Session 4: free papers \\ OC-001 INFLAMMATORY BOWEL DISEASE NURSING: RESULTS OF AN AUDIT EXPLORING THE ROLES, RESPONSIBILITIES AND ACTIVITY OF NURSES WITH SPECIALIST/ADVANCED ROLES WITHIN INFLAMMATORY BOWEL DISEASE}

doi:10.1136/gutjnl-2012-302514a.1

\begin{abstract}
${ }^{1} \mathrm{I}$ Mason, ${ }^{*}{ }^{2} \mathrm{~K}$ Holbrook, ${ }^{3} \mathrm{~V}$ Garrick, ${ }^{4} \mathrm{~K}$ Johns, ${ }^{5} \mathrm{M}$ Kane, ${ }^{6} \mathrm{~K}$ Kemp. ${ }^{1}$ Department of Gastroenterology, Royal Free Hospital, London, UK; ${ }^{2}$ Department of Gastroenterology, Gloucestershire Royal Hospital, Glouchester, UK; ${ }^{3}$ Department of Gastroenterology, Royal Hospital for Sick Children, Glasgow, UK; ${ }^{4}$ Department of Gastroenterology, Hywel Dda NHS Trust, UK; ${ }^{5}$ Department of Gastroenterology, Northern Trust, UK; ${ }^{6}$ School of Nursing, Department of Gastroenterology, University of Manchester, Manchester Royal Infirm, Manchester, UK
\end{abstract}

Introduction The role of the nurse specialist in inflammatory bowel disease (IBD) in the UK has developed significantly. Audit work has illustrated the quality indicators provided by the role yet systematic review identified the need for clearer role definition and the completion of a scoping exercise of posts and models of practice. This abstract provides the results of the first UK national audit of IBD nursing.

Methods Each UK country was represented in the dataset, adults and paediatrics. Inclusion criteria: nurse with a specialist/advanced role caring for patients with IBD. Nurses were identified through the Royal College of Nursing IBD Network and advertising at meetings/ nursing press. The audit was in three parts: demographic of the IBD nurse; demographic of IBD nurse service; prospective individual nurse activity over a 10-day period. An electronic reporting system was used to enter data and completed during a 10-day survey period May 2011. Results Response rate was 82.5\% (198/240). Numbers of IBD nurses are increasing but fall short of the recommended level set by the IBD Standards. Half of the nurses had been employed for $<5$ yrs $(54 \%)$ and were in their first specialist nursing role. A quarter (25.8\%) held no formal qualification at degree level. 55\% make decisions to prescribe, drug type, dosage and treat. Over the 10-day audit period 6472 calls were taken through telephone advice lines, 3256 patients were seen in outpatient clinics, 112 in-patients with IBD were reviewed by the nurse specialists and 1203 patients attended to the nursing service for day case treatment. Services provided included education, telephone, inpatient/outpatient follow-up and management of biologic services. Advanced nursing practice, nurse led clinics and non-medical prescribing was identified. Two thirds of IBD nurse specialist services are suspended when the IBD nurse is away. Extrapolating daily over a 12 month period the data shows IBD nurses provide 28980 patient episodes per year and work on average 33 min extra per day, equates to a minimum $£ 347200$ per year.

Conclusion IBD nurses in the UK are carrying out significant levels of activity influencing the management of considerable numbers of patients and play a fundamental role in complex medicine management. Roles are exclusively based in acute care and have other GI nursing roles alongside IBD. Recommendations are a review of education and training needs and further national audit of the quality of patient experience in relation to IBD nursing services.

Competing interests None declared.

\section{OC-002 TEAM SIMULATION TRAINING FOR MEDICAL EMERGENCIES IN THE ENDOSCOPY UNIT}

doi:10.1136/gutjnl-2012-302514a.2

S Ray, ${ }^{*}$ K Hills. Department of Gastroenterology, Queen Elizabeth the Queen Mother Hospital, Margate, UK

Introduction Medical simulation is as an attractive way to practice clinical skills to improve confidence, teamwork and outcomes. ${ }^{1}$ The endoscopy unit is an arena in which a heterogenous group of patients present with complex medical comorbidities. While emergencies are uncommon, they require prompt \& seamless management. Simulation is common in industries such as aeronautics to practice rare but predictable incidents. Simulation is also used for teaching endoscopy. We therefore piloted novel simulation training in medical emergencies for our own unit's endoscopy staff (ES) before rolling this out across our trust in East Kent.

Methods We conducted three half-day sessions in a dedicated simulation complex. ES were divided into teams of two nurses and a healthcare assistant rotating through scenarios such as anaphylaxis and angina followed by feedback, tutorials \& literature. The scenario room was equipped with a sophisticated mannequin capable of speech \& respiration attached to monitoring including pulse, oxygen saturations \& blood pressure manipulated from a control room. The room was fitted with cameras and microphones to assist feedback. There was a viewing chamber in which ES could watch their colleagues. Written candidate feedback was obtained.

Results In April 2010, six of our ES completed the pilot session. In September 2010 \& 2011, 12 ES from two hospitals in our trust completed the session. None of the ES had ever had specific training in this area and were not confident in their abilities prior to the session. The vast majority found the training to be enjoyable, useful and that it enhanced their confidence.

Conclusion Simulation is becoming recognised as an important training tool in rehearsing responses to a variety of clinical settings. Our experience demonstrates that our ES did not feel confident in recognising and managing medical emergencies perhaps as they do not encounter them as frequently as ward nurses. The absence of such training is an omission in improving patient safety. The use of training in teams which work together on a daily basis helped reduce anxiety and improved confidence and the overall realism of the simulation. The staff strongly felt that this training was realistic and targetted to their learning needs and experience. While the use of our sophisticated simulation facility was advantageous in running our programme this is not critical to propagating this to other trusts. The mannequin and much of the equipment is portable and our next aim is to bring the simulation inside the endoscopy room to enhance realism and facilitate transfer to any endoscopy unit.

Competing interests None declared.

\section{REFERENCE}

1. Ruesseler $\mathbf{M}$, Weinlich M, Müller MP, et al. Simulation training improves ability to manage medical emergencies. Emerg Med J 2010;27:734-8.

\section{OC-003 NURSE VS DOCTOR CONSENT WITHIN ENDOSCOPY: ARE THEY EQUAL?}

doi:10.1136/gutjnl-2012-302514a.3

J Waloszkova, ${ }^{*}$ F Avey, A Leahy. Endoscopy Unit, West Hertfordshire Hospitals NHS Trust, Watford, UK

Introduction This study compared the effectiveness of nurse led consent vs doctor led consent for patients undergoing day case diagnostic endoscopy. There have been no previous studies that have directly compared nurse to doctor consent within endoscopy. Ten years ago, it was suggested that consent at that time was mostly a doctor's task, but it might be beneficial to involve a nurse in the consent process. ${ }^{1}$ There is a need to determine whether nurse consent is as effective as doctor consent, as nurse consent could lead to a more streamlined patient service, improve unit efficiency and increase job satisfaction.

Methods The study assessed patient knowledge, understanding and experience of an endoscopic procedure after being consented by either a nurse or a doctor. Patients who had a diagnostic gastroscopy 
were prospectively studied. The following groups of patients were excluded: non-english speaking patients; patients with previous experience of the procedure; sedated patients as the sedation might have impaired mental recall: and inpatients because of possible confounding comorbidity. An abbreviated previously validated consent questionnaire was used (the OuIC questionnaire), ${ }^{2}$ with specific reference to the overall understanding of the test, risk/ benefits and alternatives. The significance of observed differences were statistically assessed by the Student's t-test.

Results Patients were consented by either their named nurse or the doctor carrying out the procedure. All nurses trained in consent participated in this study, 10 staff nurses and two sisters. Doctors who participated in this study were four consultant gastroenterologists, three gastroenterology registrars, two consultant surgeons and one elderly care consultant. Questionnaires were completed for 65 nurse consent and 47 doctor consents. Patients had a better understanding of the alternatives to having the procedure when consented by a nurse $(p<0.03)$. Patients understood the risks, discomfort, benefits and the overall procedure equally whether consented by a nurse or doctor.

Conclusion This study has shown that nurse consent in our unit was more thorough than doctor consent. Patients who were consented by a nurse showed greater understanding of the alternative options to having the endoscopy. There was equal understanding of the risks, discomfort, benefits and overall procedure whether consented by a nurse or a doctor. Based on these results, endoscopy units should consider adopting nurse led consent into their daily working practice.

Competing interests None declared.

\section{REFERENCE}

1. Joffe S, Cook EF, Cleary EF, et al. Quality of informed consent: a new measure of understanding among research subjects. J Natl Cancer Inst 2001:93:193-47.

2. McParland J, Scott PA, Arndt M, et al. Autonomy and clinical practice 3: issues of patient consent. Br J Nurs 2000;9:660-5

\section{OC-004 ASSISTANT PRACTITIONERS IN ENDOSCOPY: INNOVATIVE PRACTISE}

doi:10.1136/gutjnl-2012-302514a.4

V Jackson, H Murray. * Department of Endoscopy, Aintree Hospitals NHS Foundation Trust, Liverpool, UK

Introduction New roles are being introduced within the NHS to utilise knowledge and skills to offer value for money services. Assistant Practitioners were introduced into the endoscopy unit and an innovative service was set up to improve the patient management for pre surveillance colonoscopy screening.

Methods A telephone assessment was implemented by an Assistant Practitioner (band 4) to ascertain whether this could be offered as an alternative to the current pre endoscopy clinic led by a band 5 to ensure the patient was safe to proceed with bowel preparation and investigation.

Results 68 patients were booked onto the Assistant Practitioner (AP) telephone clinic over a 10-week period. Out of these 20 DNA'd the appointment and 48 were assessed via the telephone consultation. Out of the 48 patients assessed the AP was able to decide if the patient was fit to proceed without seeking further advice. Forty-one patients were deemed suitable to advance to colonoscopy and agreed a date to attend. Seven patients chose to be discharged.

Conclusion Hospital attendance is not always required to adequately assess the suitability for bowel preparation and colonoscopy. For a cohort of patients this can be done by a telephone consultation. This has advantages both for the user, in that they are not required to attend with all the difficulties associated with hospital visits, and the provider for adequate use of resource and capacity available. It is acknowledged that there may be a proportion of patients identified that require a face to face assessment at a higher level and those can be given a date to attend a nurse led clinic at the time of telephone consultation. This method is more cost effective due to the higher numbers of consultations on a telephone assessment clinic compared to face to face clinic and the associated salaries of the band of the practitioner undertaking the session.

Competing interests None declared.

\section{OC-005 AN EXPLORATION OF THE HEALTH AND SOCIAL NEEDS OF PEOPLE LIVING WITH INFLAMMATORY BOWEL DISEASE: A METASYNTHESIS}

doi:10.1136/gutjnl-2012-302514a.5

${ }^{1} \mathrm{~K}$ Kemp, ${ }^{2} \mathrm{~J}$ Griffiths, ${ }^{2} \mathrm{~K}$ Lovell. ${ }^{1}$ School of Nursing, Department of Gastroenterology, University of Manchester, Manchester Royal Infirm, UK; ${ }^{2}$ School of Nursing, University of Manchester, Manchester, UK

Introduction The Inflammatory Bowel Disease Questionnaire and the Rating Form for IBD patient's Concerns, are widely used measures in IBD but fail to capture the essence of living with IBD from the patient's perspective. To deliver patient centred care it is imperative that we have an understanding of the experience of living with IBD. This study aimed to synthesise the findings of qualitative papers that explored the health and social needs of patients living with IBD.

Methods A systematic literature search was conducted in MEDLINE, PsychInfo, EMBASE, CINAHL, British Nursing Index via the OVID platform to August 2010. Search filters developed by the Hedges database, McMaster University Health Information Research Unit and Kathryn Nesbit, Edward G Milner Library, University of Rochester, were adapted to aid the search. 1395 papers were generated by the preliminary search. Qualitative studies which explored the phenomena of living with IBD, English language and sample population adults $>16$ years were included. Study eligibility judgements and data extraction were independently completed by the authors. The papers were quality appraised using the Critical Appraisal Skills Programme. Synthesis was achieved by comparing the themes and findings of each study with one another to identify 1st order constructs. Repeated comparison between the papers revealed the similarities and differences, which led to 2 nd order constructs and the new interpretation of the synthesised studies.

Results Six papers and one unpublished thesis were included, all from Western countries, one study included patients from an immigrant background. Combined sample of the studies was only 86 patients to describe the phenomena of living with IBD. First iteration of synthesis identified 16 themes, 2nd iteration synthesised these into three main constructs: "detained by the disease"; "living in a world of disease"; "wrestling with life". "Detained by the disease" is the fear of incontinence, and the behaviour the patients displays as a result of this. Social isolation and missing out on life events all serve to "pull" the patient back from normal living. "Living in a world of disease" is living with the fear of a long term condition." Wrestling with life" is the "push" to continue normal living. Conclusion People with IBD endure many daily challenges, stress, pain, fighting for control. The combined impact of living with IBD is the tension they live with. The value of metasynthesis is the interpretation of all of the synthesised studies to provide a global representation of living with IBD: "Pushed and pulled: a compromised life", people living with IBD experience a constant conflict throughout their lives, they push to be normal but IBD pulls them back

Competing interests None declared. 